Case Report

\title{
Clostridium cadaveris Osteomyelitis: an Unusual Pathogen which Highlights the Importance of Deep Tissue Sampling in Chronic Osteomyelitis
}

\author{
Ruth Alexandra Corrigan ${ }^{1,2}{ }^{\bowtie}$, Jose Lomas-Cabeza ${ }^{1}$, David Stubbs ${ }^{1}$, Martin McNally ${ }^{1}$ \\ 1. Bone Infection Unit, Nuffield Orthopaedic Centre, Oxford University Hospitals NHS Foundation Trust, Windmill Road, Oxford, OX3 7HE, UK. \\ 2. Nuffield Department of Clinical Laboratory Sciences, Radcliffe Department of Medicine, John Radcliffe Hospital, Headley Way, Oxford, OX3 9DU, UK. \\ $\triangle$ Corresponding author: Ruth Alexandra Corrigan, E-mail: ruth.corrigan@doctors.org.uk. Telephone: 07932 514595, Nuffield Department of Clinical \\ Laboratory Sciences, Radcliffe Department of Medicine, John Radcliffe Hospital, Headley Way, Oxford, OX3 9DU. \\ ( $)$ The author(s). This is an open access article distributed under the terms of the Creative Commons Attribution License (https://creativecommons.org/licenses/by/4.0/). \\ See http://ivyspring.com/terms for full terms and conditions.
}

Received: 2020.01.09; Accepted: 2020.02.23; Published: 2020.03.30

\begin{abstract}
Clostridium cadaveris, named following its identification in human corpses, is an unusual pathogen. We report the first case of $C$. cadaveris osteomyelitis. This case highlights the importance of deep tissue sampling and appropriate culture to correctly identify causative pathogens and guide targeted antimicrobial therapy in difficult-to-treat infections like chronic osteomyelitis.
\end{abstract}

Key words: Chronic osteomyelitis, intra-operative sampling, Clostridium cadaveris

\section{Background}

The genus Clostridium contains over 220 obligate anaerobic (occasionally aerotolerant species) most of which are considered clinically insignificant environmental bacteria. C. cadaveris is usually a nonpathogenic and non-toxin producing, enteric, gasforming anaerobe. First described by Klein in 1899 as the most prominent bacteria present during human decomposition [1], it is found in both the human gut and the environment.

Bacteraemia with Clostridia is rare, calculated to be responsible for $0.5 \%-2 \%$ of all positive anaerobic blood cultures in one study [2]. Infections are most often gastrointestinal in origin [2,3] and affect those with predisposing immunosuppression (malignancy, diabetes, alcohol abuse, steroids, chemotherapy) [3-6]. There are approximately 30 cases of Clostridial osteomyelitis published to date (Table 1 [7-25]). Of note, since 2016, Clostridioides difficile (previously Clostridium difficile) is considered a different genus based on phenotypic, chemotaxonomic and phylogenetic analysis [26] and so is not considered further here. Over 30 cases of clostridial septic arthritis have also recently been summarised [27]. Osteomyelitis due to clostridial species usually follows trauma [14] or open fractures [7,8,11,12,15-18], some obviously contaminated with soil $[12,15,17]$ or sewerage $[8,11]$. In non-traumatic cases hosts may be immunocompromised by lymphoma $[13,22]$ or diabetes [10], though sometimes there is neither a clearly identifiable source nor host risk factor [9]. Nine of these cases have been reported in the last five years which likely reflects laboratory advances in diagnostic capabilities including improved anaerobic culture techniques and the increasing availability of MALDITOF mass spectrometry and genetic sequencing for exact species determination.

Although there are some reports of nonorthopaedic infections in immunocompetent individuals [28] this is the first case report of $C$. cadaveris osteomyelitis in either immunocompromised or healthy individuals.

Chronic osteomyelitis can be a notoriously difficult infection to diagnose and treat. A high clinical index of suspicion, appropriate imaging, 
thorough debridement and long-term targeted antibiotic therapy is widely considered the best management strategy. Thorough tissue sampling and appropriate culture is essential to identify causative organisms to permit targeted antibiotic therapy and effective treatment $[29,30]$.

\section{Case presentation}

A 32 year old man presented to us with a 12 month history of infection around his lower right tibia and ankle. There was no history of fracture; rather, the infection was attributed to a soft tissue injury sustained after impact with a concrete bollard whilst running. Before presentation to us he had undergone incision and drainage of a large volume of pus as well as windowing of the tibia and two bony debridement procedures, the last 6 months previously. A wound swab taken at the time of injury had grown a fully sensitive Group A haemolytic streptococcus, and a single intraoperative sample from the right distal tibia taken at the time of the last debridement had grown Staphylococcus aureus (resistant to erythromycin). Blood cultures had remained negative throughout. He had completed a short course of oral clindamycin 300mg QDS (5 days) and oral flucloxacillin 500mg QDS prescribed by his GP at the time of initial presentation, as well as a second short course of oral flucloxacillin and two short courses of oral amoxicillin immediately after each of his surgical procedures. He was a smoker, but had no other significant medical co-morbidities.

At the time of our assessment he was afebrile and systemically well, though complained of ongoing ankle discomfort and swelling. On examination there was a scar over the anteromedial border of the tibia, with a small amount of skin discolouration and dry crust over the lower third. He had strong pedal pulses, normal sensation and a good range of ankle movement. Pre-operative imaging was consistent with Cierny and Mader Anatomic Type 3 osteomyelitis of the right lower tibia (Figure 1).

He was taken to theatre for surgical excision, deep tissue sampling and reconstruction. The previous bone window was extended and the cavity was curetted back to healthy bleeding bone. Five deep samples were taken using a validated sampling protocol [31,32]. Briefly this protocol recommends that when suspecting infection, 5 or more deep samples are taken with minimal manipulation of the target area using separate, unused instruments for each sample. Preferably samples should be taken prior to administration of intra-operative antibiotics, and after withholding antibiotics for at least 2 weeks prior to sampling. No pus was seen, but a significant area of dead infected bone was removed leaving a cortico-medullary bone defect. The resection site was washed with $0.05 \%$ aqueous chlorhexadine and the bone defect was filled with gentamicin eluting, bioabsorbable composites (Herafil ${ }^{\circledR}$ beads $G$ [Heraeus Medical] and Cerament ${ }^{\mathrm{TM}} \mathrm{G}$ (20mls) [Bone Support]). The soft-tissue defect was reconstructed with a free gracilis muscle flap and split skin graft (Figure 2). Post operatively he was treated empirically for five days with vancomycin $1 \mathrm{~g}$ BD and meropenem 500mg TDS.
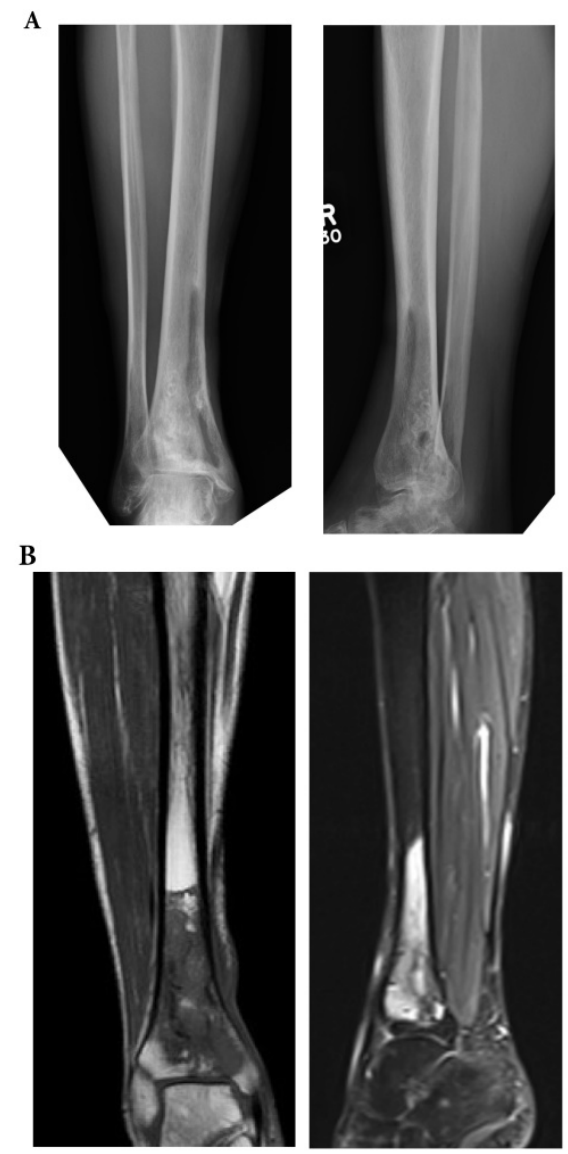

Figure 1. Pre-operative radiograph (a) and MRI (b) suggestive of Cierny and Mader Stage 3 osteomyelitis of the lower tibia.
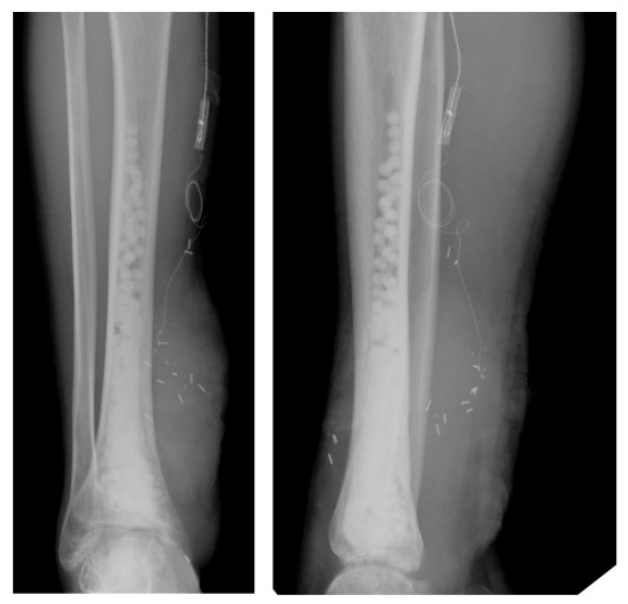

Figure 2. Post-operative radiographs with gentamicin eluting, bioabsorbable composites visible at the site of osteomyelitis excision. 
Table 1. A summary of published cases of Clostridial osteomyelitis

\begin{tabular}{|c|c|c|c|c|}
\hline Paper & Clostridium species & Type of infection & No. of cases & Case details (where known) \\
\hline $\begin{array}{l}\text { Mormeneo Bayo et al. } \\
2020\end{array}$ & Clostridium celerecrescens & Fracture related infection & 1 & $\begin{array}{l}39 \mathrm{~F} \text { sustained bilateral open femoral fractures in } \\
\text { road traffic accident }\end{array}$ \\
\hline \multirow[t]{2}{*}{ Tremp et al. 2020} & Clostridium spp. & Fracture related infection & 1 & $\begin{array}{l}43 \mathrm{~F} \text { farmer stumbled over doorstep, sustained } \\
\text { trimalleolar open fracture }\end{array}$ \\
\hline & Clostridium butyricum & Fracture related infection & 1 & $\begin{array}{l}58 \mathrm{~F} \text { fell into cesspool, sustained bimalleolar fracture } \\
\text { and soft tissue injury }\end{array}$ \\
\hline $\begin{array}{l}\text { Vijayvargiya et al. } \\
2019\end{array}$ & Clostridium paraputrificum & Septic arthritis and osteomyelitis & 1 & $\begin{array}{l}\text { 86F native shoulder joint septic arthritis and } \\
\text { osteomyelitis }\end{array}$ \\
\hline Abusnina et al. 2019 & Clostrodium sporogenes & Osteomyelitis & 1 & $\begin{array}{l}66 \mathrm{~F} \text { obese non-insulin dependent diabetic with } \\
\text { pressure ulcer associated osteomyelitis }\end{array}$ \\
\hline Hirai et al. 2017 & Clostridium hydrogeniformans & Fracture related infection & 1 & $\begin{array}{l}\text { 18M sustained open right arm fracture in motorbike } \\
\text { accident contaminated by drain contents }\end{array}$ \\
\hline Perkins et al. 2017 & Clostridium sphenoides & Fracture related infection & 1 & $\begin{array}{l}\text { 20M sustained open right radius and ulna fracture } \\
\text { whilst wrestling, contaminated with soil. }\end{array}$ \\
\hline Mutoh et al. 2015 & Clostridium innocuum & Osteomyelitis & 1 & $\begin{array}{l}\text { 32M with ALL and pelvic osteomyelitis and } \\
\text { bacteraemia, unclear source }\end{array}$ \\
\hline Virot et al. 2015 & Clostridium tertium & Osteomyelitis & 1 & $\begin{array}{l}40 \mathrm{M} \text { ex soldier with osteomyelitis surrounding } \\
\text { shrapnel in tibia }\end{array}$ \\
\hline $\begin{array}{l}\text { Ibnoulkhatib et al. } \\
2012\end{array}$ & Clostridium spp. & Fracture related infection & 12 & $\begin{array}{l}\text { Multiple cases of traumatic fracture, each with soil } \\
\text { contamination }\end{array}$ \\
\hline Mischnik et al. 2011 & Clostridium celerescens & Fracture related infection & 1 & $\begin{array}{l}55 \mathrm{M} \text { with peripheral vascular disease and a history } \\
\text { of open tibial fracture }\end{array}$ \\
\hline Taylor et al. 2010 & Clostridium argentinense & Fracture related infection & 1 & $\begin{array}{l}34 \mathrm{M} \text { with contaminated open fracture of right ulna } \\
\text { and radius playing soccer }\end{array}$ \\
\hline Jiang et al. 2009 & Clostridium glycolicum & Fracture related infection & 1 & $\begin{array}{l}20 \mathrm{~F} \text { with open fracture of radius and ulna after a } \\
\text { road vehicle accident }\end{array}$ \\
\hline Tekaya et al. 2008 & Clostridium clostridiiforme & Vertebral osteomyelitis & 1 & \\
\hline Kihiczak et al. 1999 & Clostridium septicum & Osteomyelitis & 1 & \\
\hline Shetty et al. 1998 & Clostridium septicum & Osteomyelitis & 1 & \\
\hline Scanlan et al. 1994 & Clostridium bifermentans & Osteomyelitis & 1 & $\begin{array}{l}\text { 81M with oropharyngeal lymphoma, bacteraemia } \\
\text { and multifocal osteomyelitis (T11-L5, sacrum and } \\
\text { ribs) of unclear source }\end{array}$ \\
\hline Spitzer et al. 1991 & Clostridium clostridiiforme & Osteomyelitis & 1 & \\
\hline Brook et al. 1993 & Clostridium spp. & 'Bone infection' & 1 & \\
\hline Neimkin et al. 1985 & Clostridium septicum & Osteomyelitis & 1 & \\
\hline
\end{tabular}

A

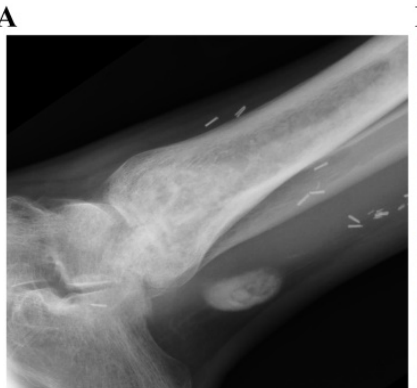

B

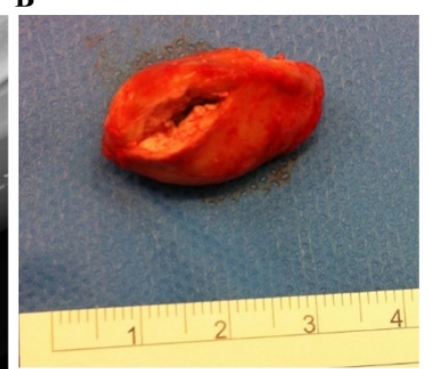

Figure 3. Radiograph (a) at 10 months showing a small radiodense area of extruded antibiotic carrier adjacent to the tibialis posterior tendon. The bone defect has remodelled well with no sign of recurrent infection. At operation, a $1 \times 2 \times 2.5 \mathrm{~cm}$ soft mass of calcium sulphate was removed (b).

In the laboratory, a preparation from each deep tissue sample was inoculated into both a BD BACTEC $^{\mathrm{TM}}$ Plus aerobic/F bottle and a BD BACTEC $^{\text {TM }}$ Lytic/10 Anaerobic/F bottle and incubated at 35-37 degrees within a BD BACTECTM FX system. After 48 hours incubation C. cadaveris grew in four out of five samples incubated under anaerobic conditions (sensitive to penicillin, tetracycline, erythromycin, fusidic acid, rifampicin, linezolid, vancomycin and metronidazole, as assessed by disc diffusion). No other organisms (including further
Staphylococci or Streptococci) were isolated. Deep tissue histology was consistent with active chronic infection, but no organisms were seen with Gram stain. He was commenced on a 3 month course of oral clindamycin $450 \mathrm{mg}$ TDS and discharged on postoperative day ten.

\section{Outcome and follow up}

At follow up 6 weeks later the wound was healed and the patient was systemically well. At 9 months after surgery, he still complained of ongoing pain and stiffness behind the medial malleolus. This was due to irritation of his tibialis posterior tendon by a small collection of calcium sulphate extruded from his bone defect (Figure 3A). He underwent excision of this without complication (Figure 3B). He was discharged from our care 21 months after his initial surgery, fully mobile and with no evidence of ongoing osteomyelitis.

\section{Discussion}

This is the first published case of C. cadaveris osteomyelitis. That this unusual pathogen was isolated only at the time of definitive surgery 
following multiple failed therapeutic strategies highlights the importance of robust deep tissue sampling in the management of chronic osteomyelitis. It is likely that this infection was caused by direct inoculation following a contaminated soft tissue injury. There were no other identifiable host risk factors.

With all chronic infections it is essential to identify the causative organism to permit targeted antibiotic treatment, both to help prevent the development of multi-resistant pathogens and to contain infections in a timely manner. In this case, $C$. cadaveris had not been isolated from any samples taken prior to this most recent osteomyelitis excision. However, all of the cultures of deep wound samples taken previously had been inadequate: none had been cultured anaerobically. It is imperative, with chronic infections not responding to empirical or even targeted therapy, to ensure that appropriate cultures for fastidious organisms are completed. It is likely that C. cadaveris persisted despite the previous antibiotic courses due to incomplete excision as well as sub optimal duration of antibiotic therapy and poor penetrance of antibiotics to the site of infection.

Our decision to use a long course of clindamycin to treat this multi-sensitive pathogen was primarily based upon a concern regarding the possibility of an incomplete excision. Oral clindamycin has good bone penetrance and good activity against anaerobic bacteria. Concerns about pseudomembranous colitis are less immediate in a young, otherwise healthy man. Co-amoxiclav or metronidazole could have been suitable alternatives but the poor bone penetrance of oral beta lactams plus the side effect profile of metronidazole make these less favourable choices for a long term antibiotic course.

In summary, this case highlights the need for multiple deep tissue samples with appropriate culture to identify unusual causative organisms in chronic deep tissue infections. This is the first case of $C$. cadaveris osteomyelitis in the literature, identified only after suitable culture technique was employed. Identification of the causative pathogen enabled targeted antibiotic therapy which contributed to the overall successful outcome.

\section{Learning points}

- C. cadaveris is an unusual anaerobic pathogen and previously unreported as a causative agent of osteomyelitis.

- Careful, fully sterile and repeated intra-operative sampling is essential for effective and targeted antibiotic treatment of chronic osteomyelitis.

- Anaerobic and aerobic cultures are essential to identify all relevant potentially pathogenic organisms.

- Antibiotic choice in chronic osteomyelitis should take into account extent of excision, organism sensitivities and antibiotic penetrance, mechanism of action and side effect profile, especially when long courses are indicated.

\section{Abbreviations}

ALL: acute lymphocytic leukaemia; MALDITOF: Matrix-Assisted Laser Desorption/IonizationTime of Flight; BD: bis die (twice daily); TDS: ter die sumendum (three times daily); QDS: quater die sumendus (four times daily); GP: general practitioner.

\section{Acknowledgments}

With thanks to the clinical and laboratory staff involved in this case.

\section{Authors Contributions}

RAC and MM wrote the manuscript, JLC determined the antibiotic plan; MM and DS planned and performed the operations. All authors reviewed the manuscript.

\section{Competing Interests}

The authors have declared that no competing interest exists.

\section{References}

1. Klein E. Ein Beitrag zur Bakteriologie der Leichenverwesung. Zentralblatt für Bakteriologie, Parasitenkunde, Infektionskrankheiten und Hygiene 1899; 1: 278-84.

2. Brook I. Anaerobic bacterial bacteremia: 12-year experience in two military hospitals. J Infect Dis. 1989; 160: 1071-5.

3. de Virgilio C, Klein S, Chang L, et al. Clostridial bacteraemia: implications for the surgeon. Am Surg. 1991; 57(6): 388-93.

4. Herman R, Goldman IS, Bronzo R, et al. Clostridium cadaveris: an unusual cause of spontaneous bacterial peritonitis. Am J Gastroenterol. 1992; 87(1): $140-2$

5. Gucalp R, Motyl M, Carlisle P, et al. Clostridium cadaveris bacteremia in the immunocompromised host. Med Pediatr Oncol. 1993; 21(1): 70-2.

6. Morshed S, Malek F, Silverstein RM, et al. Clostridium cadaveris septic arthritis after total hip arthroplasty in a metastatic breast cancer patient. J Arthroplasty. 2007; 22(2): 289-282.

7. Mormeneo Bayo S, Ferrer Ceron I, Martín Juste P et al. A review of difficult-to-treat post-traumatic osteomyelitis: Role of Clostridium celerecrescens. Rev Esp Cir Ortop Traumatol. 2020; [Epub ahead of print].

8. Tremp M, Oranges CM, Majewski M et al. Clostridial Infection After Open Fractures of the Lower Extremity - Report of Two Cases and Discussion of Pathomechanism and Treatment. In vivo. 2020; 34(1): 291-298.

9. Vijayvargiya P, Garrigos ZE, Rodino KG et al. Clostridium paraputrificum septic arthritis and osteomyelitis of shoulder: A case report and review of literature. Anaerobe. 2019. doi: 10.1016/j.anaerobe.2019.102105.

10. Abusnina $\mathrm{W}$, Shehata $\mathrm{M}$, Karem $\mathrm{E}$ et al. Clostridium sporogenes bacteremia in an immunocompetent patient. ID Cases. 2019; 15: e00481. doi:10.1016/j.idcr.2018.e00481.

11. Hirai J, Sakanashi D, Huh JY et al. The first human clinical case of chronic osteomyelitis caused by Clostridium hydrogeniformans. Anaerobe. 2017; 45: 138-141.

12. Perkins MJ, Snesrud E, McGann P et al. Clostridium sphenoides Chronic Osteomyelitis Diagnosed Via Matrix-Assisted Laser Desorption Ionization Time of Flight Mass Spectrometry, Conflicting With 16S rRNA Sequencing but Confirmed by Whole Genome Sequencing. Military Medicine. 2017; 182(1-2): e1669-e1672. 
13. Mutoh Y, Hirai R, Tanimura A et al. Osteomyelitis due to Clostridium innocuum in a patient with acute lymphoblastic leukemia: case report and literature review. Springerplus. 2015; 4:385. doi:10.1186/s40064-015-1176-3.

14. Virot E, Servien E, Laurent F et al. Reactivation of Clostridium tertium bone infection 30 years after the Iran-Iraq war. Case Reports. 2015; 2015:bcr2014209169.

15. Ibnoulkhatib A, Lacroix J, Moine A et al. Post-traumatic bone and/or joint limb infectons due to Clostridium spp. OTSR. 2012; 98(6): 696-705.

16. Mischnik A, Zimmermann S, Bekeredjian-Ding I et al. Relapse of posttraumatic osteomyelitis due to Clostridium celerecrescens. Infection. 2011; 39: 491-494.

17. Taylor SM, Wolfe CR, Dixon TC et al. Wound botulism complicating internal fixation of a complex radial fracture. J Clin Microbiol. 2010; 48 (2): 650-653.

18. Jiang W, Abrar S, Romagnoli $\mathrm{M}$ et al. Clostridium glycolicum wound infections: case reports and review of the literature. J Clin Microbiol. 2009; 47 (5): 1599-1601.

19. Tekaya R, Saidane $\mathrm{O}$, Abdelmoula $\mathrm{L}$ et al. Spondylodiscite à Clostridium clostridiiforme. Med Mal Infect . 2008; 38 (6): 332-33.

20. Kihiczak D, Epstein R. Clostridium septicum osteomyelitis with intramedullary gas on CT and MR imaging. AJR Am J Roentgenol. 1999; 172: $1457-1458$.

21. Shetty AK, Heinrich SD, Steele RW. Clostridium septicum osteomyelitis: case report and review. Pediatr Infect Dis J. 1998; 17(10): 927-9.

22. Scanlan DR, Smith MA, Isenberg HD et al. Clostridium bifermentans bacteremia with metastatic osteomyelitis. J Clin Microbiol. 1994; 32(11): 2867-2868.

23. Spitzer RD and Ratzan KR. Chronic osteomyelitis due to Clostridium clostridiiforme. South Med J. 1991; 84: 671-672.

24. Brook I and Frazier EH. Anaerobic osteomyelitis and arthritis in a military hospital: A 10-year experience. Am J Med. 1993; 1: 21-28.

25. Neimkin RJ and Jupiter JB. Metastatic nontraumatic Clostridium septicum osteomyelitis. J. Hand Surg. 1985: 1OA; 281-283.

26. Lawson PA, Citron DM, Tyrrell KL et al. Reclassification of Clostridium difficile as Clostridioides difficile (Hall and O'Toole 1935) Prévot 1938. Anaerobe. 2016; 40: 95-99.

27. Gredlein CM, Silverman ML, Downey MS. Polymicrobial septic arthritis due to Clostridium species: case report and review. Clin Infect Dis. 2000: 30(3); 590-4.

28. Poduval RD, Mohandas R, Unnikrishnan, D et al. Clostridium cadaveris bacteremia in an immunocompetent host. Clin Infect Dis. 1999; 29(5): 1354-1355.

29. Sheehy SH, Atkins BA, Bejon $\mathrm{P}$, et al. The microbiology of chronic osteomyelitis: Prevalence of resistance to common empirical anti-microbial regimens. J. Infect 2010; 60: 338-343.

30. McNally M and Nagarajah K. Osteomyelitis. Orthop Trauma. 2010; 24(6): 416-429.

31. Sigmund IK and McNally MA. Diagnosis of bone and joint infections. Orthop Trauma. 2019; 33(3): 144-152.

32. Govaert GAM, Kuehl MD, Atkins BL et al. Diagnosing Fracture-Related Infection: Current concepts and recommendations. Orthop Trauma. 2020; 34(1): 8-17. 